\title{
INCIDÊNCIA E ASPECTOS HISTOPATOLÓGICOS DA INFECÇÃO POR HELICOBACTER SPP. EM GATOS DA CIDADE DE RECIFE, PERNAMBUCO, BRASIL
}

\author{
J.S. Macêdo*, F.S. Mendonça, K.R.L. da Silva, M.E.G. de Barros, J. Evêncio-Neto
}

Universidade Federal Rural de Pernambuco, Departamento de Morfologia e Fisiologia Animal, Rua Dom Manoel de Medeiros s/no, CEP 52171-900, Recife, Brasil. E-mail: evencioneto@pq.cnpq.br

\section{RESUMO}

\begin{abstract}
O presente trabalho objetivou investigar a prevalência da bactéria Helicobacter spp. em gatos da Cidade do Recife, Pernambuco. As amostras de estômagos analisadas foram colhidas de 119 gatos sem raça e idade definida, sendo 56 machos e 63 fêmeas. Para pesquisa de Helicobacter spp., foram realizados os métodos de teste rápido da urease e exames histopatológicos. As análises pelo teste rápido da urease mostraram que em $82,35 \%$ das amostras foi detectada reação positiva para Helicobacter. Na análise histopatológica, verificou-se que em 59,66\% das amostras (71/119) apresentaram lesões. Essas lesões consistiram em infiltrado linfoplasmocitário com folículo linfoide, microabcessos, necrose, hipotrofia; em 40,34\% das amostras (48/119) não foram observadas alterações histológicas. Conclui-se que gatos oriundos da Cidade de Recife, Pernambuco< apresentam alta incidência de infecção por Helicobacter spp. Infiltrado difuso de células linfoplasmocitárias foram as alterações histopatológicas mais frequentes observadas na mucosa gástrica de gatos infectados por Helicobacter spp.
\end{abstract}

PALAVRAS-CHAVE: Helicobacteriose, urease, gastrite, bactéria, felinos.

\begin{abstract}
INCIDENCE AND PATHOLOGICAL ASPECTS OF INFECTION BY HELICOBACTER SPP. IN CATS FROM THE CITY OF RECIFE, PERNAMBUCO, BRAZIL. The present study aimed to investigate the incidence of the bacterium Helicobacter spp. in cats from Recife, Pernambuco State, Brazil. Stomach samples were collected from 119 cats, without distinction between race or age, the breakdown by sex being 56 males and 63 females. The urease quick test and histopathological examination using both hematoxilin-eosin (HE) and Giemsa stain were performed. The urease quick test showed positive results for Helicobacter in $82.35 \%$ of the samples. The histopathology revealed an alteration in 59.66\% (71/119) of the samples, such as lymphoplasmocitic infiltration with a lymphoid foliculum, microabscess, necrosis, and hypotrophy, while in $40.34 \%$ of the samples $(48 / 119)$ no alterations were observed. It was concluded that the cats from the city of Recife, State of Pernambuco, Brazil, presented a high incidence of infection with Helicobacter spp. Diffuse infiltrate of lymphocytes cells was the most frequent histopathological change observed in the gastric mucosa of cats infected with Helicobacter spp.
\end{abstract}

KEY WORDS: Helicobacteriosis, urease, gastritis, bacterium, cats.

\section{INTRODUÇÃO}

Bactérias do gênero Helicobacter têm sido observadas desde o final do século XIX (Bizzozero, 1893), infectando o estômago de humanos e espécies animais como cães, gatos, furões, suínos, algumas espécies de macacos (Hermanns et al., 1995), tigres e onça parda (CATTOLI et al., 2000).

A importância dessa bactéria tem sido descrita na patogenia da gastrite, da úlcera gástrica e duodenal (BlASER, 1990; COVER; BLASER 1995; QueIROZ et al.,1998) e, mais recentemente, como agente indutor do carcinoma gástrico no humano (MORGNER, 2000; CASTRO et al., 2003). Vários estudos têm demonstrado correlação entre a presença de Helicobaceter pylori no homem e outras doenças, como trombocitopenia autoimune, nefropatia membranosa, polineuropatias imunes agudas, doença cardíaca isquêmica (GASBARRINI; FRANCESCHI, 1999), carcinoma hepático (AvENAUD et al., 2000), colangite esclerosante primária e cirrose biliar primária (NILSSON et al., 2000), e também em doença coronariana (DANESH et al., 1999).

*M.V. Msc. Programa de Pós-Graduação em Ciência Veterinária, Universidade Federal Rural de Pernambuco (UFRPE). 
A prevalência da infecção por Helicobacter spp. em humanos difere sobretudo com a área geográfica,com a idade, com a raça e com o estado sócio econômico. De acordo com a área geográfica, a infecção mostrou ser mais prevalente em países subdesenvolvidos, numa taxa igual ou superior a 7\% de infecção, em oposição aos países industrializados, onde $40 \%$ das pessoas ou menos estão infectadas. A infecção pela bactéria tem correlação inversa com o padrão sócio-econômico e nos países em desenvolvimento, como o Brasil, a colonização do estômago humano pela H. pylori é disseminada (VAlle; Bizinelli 1993; Souto et al., 1998).

A prevalência de animais portadores com essas bactérias situa-se entre 70 e $100 \%$, demonstrando a possibilidade dos animais de companhia servirem como reservatório para a transmissão de helicobactérias aos humanos (BoyAnova et al., 2003). Em gatos, estudos demonstraram uma variação da prevalência de micro-organismos gástricos tipo Helicobacter que se situa entre 76 a 100\% (Отто et al., 1994; JalaVA et al., 1998; Neiger, 1998; Norris et al., 1999). Nessa espécie também ocorre doença gástrica que consiste em episódios intermitentes de vômito, às vezes com episódios agudos, que não responderam ao tratamento sintomático. Outros sinais não específicos incluem inapetência, anorexia, perda de peso e dor abdominal. Hematêmese e melena ocorrem se erosão eulceração gástrica ou neoplasia estiverem presentes. A diarreia é incomum, a menos que o paciente tenha doença inflamatória intestinal (TAMS, 2005).

Dois aspectos são importantes nas pesquisas envolvendo Helicobacter spp. em animais domésticos. O primeiro atenta para a possibilidade desses animais serem portadores e transmissores dos micro-organismos para os humanos (RADIN et al., 1990; HANDT et al., 1994; OtTo et al., 1994; HANDT et al.,1995; Fox et al.,1995). O segundo relaciona-se com uma perspectiva em medicina veterinária, visando à importância clínica destes micro-organismos em cães e gatos, bem como a sua funcionalidade enquanto modelos animais da infecção por helicobactérias em humanos (RADIN et al., 1990; Fox et al., 1995; HANDT et al., 1995).

Oobjetivo deste trabalhoé descrever a incidência de infecção por Helicobacter spp. na mucosa gástrica de gatos, oriundos da Cidade de Recife, Pernambuco, Brasil. Também são relatados os achados de necropsia e as alterações histopatológicas associados à essa infecção.

\section{MATERIAL E MÉTODOS}

Foram utilizados 119 estômagos de gatos sem raça definida, adultos, sendo 56 machos e 63 fêmeas, cedidos ao Laboratório de Histopatologia do De- partamento de Morfologia e Fisiologia Animal pelo Centro de Vigilância Ambiental (CVA) da Cidade de Recife.

Ao exame macroscópico do estômago analisou-se a integridade da mucosa observando-se coloração, presença de lesões pré-ulcerativas e/ou ulcerativas, alterações circulatórias, presença de parasitas eoutras alterações de superfície. Em seguida, coletaram-se fragmentos de estômago de cada animal para a realização do teste rápido de urease e exame histopatológico.

Para a realização do teste de urease utilizou-se kit comercial (Renylab Química e Farmacêutica), para detectação de H. pylori, contendo ureia e vermelho fenol. Fragmentos do estômago foram imersos em solução reagente e incubados em temperatura ambiente. $\mathrm{O}$ teste foi considerado positivo quando a solução adquiria tonalidade rosa, indicando presença da bactéria devido à liberação de amônia. A reação foi considerada positiva quando a viragem do indicador ocorreu entre 2 e 120 minutos, sendo fortemente positiva quando a viragem do indicador ocorreu entre 2 e 30 minutos. Quando a viragem do indicador ocorreu até 120 minutos, a reação foi considerada fracamente positiva e após 120 minutos as reações foram consideradas negativas.

Fragmentos de estômago (fundo, corpo e antropilórico) foram colhidos e fixados em formol a $10 \%$ tamponado com fosfato sódio a $4 \%$ por 24 horas, em seguida, foram desidratados em concentrações crescentes de álcool etílico, diafinizados pelo xilol e impregnados pela parafina líquida em estufa regulada à temperatura de $60^{\circ} \mathrm{C}$ e emblocados em parafina, segundo a metodologia preconizada por Michalany (1980). Na sequência, os blocos foram cortados em micrótomo do tipo Minot, ajustado para $4 \mathrm{~mm}$. Os cortes assim obtidos foram transferidos para lâminas histológicas e, em seguida, mantidas em estufa regulada à temperatura de $46^{\circ} \mathrm{C}$, durante 24 horas, para secagem. Quatro lâminas foram preparadas de cada animal e coradas por hematoxilina e eosina (H.E) (BARRos; MARQUES, 2003) e pela técnica de coloração do Giemsa (NELSON; Couto, 2006) para posterior descrição morfológica. Para este fim foi utilizado um microscópio de luz, da $\operatorname{mar}^{1}$ ca Carl Zeis, com objetivas variando de 4 a $100 \mathrm{X}$.

Para a análise histopatológica foram analisados os seguintes parâmetros: 1 . Análise quantitativa - presença de infiltrado inflamatório linfoplasmocitário na lâmina própria com ou sem formação de folículos linfoides; presença de neutrófilos em torno dos colos glandulares e fovéolas; perda dos componentes próprios da mucosa com rarefação e afastamento das glândulas e alargamento das fovéolas. 2. Alterações de natureza qualitativa - Alterações do epitélio superficial foveolar; degeneração, edema e congestão; presença de displasia, erosões, fibrose da 
lâmina própria, proliferação vascular e metaplasia pseudoantral (Bogliolo, 2006).

\section{RESULTADOS}

Os resultados das avaliações macroscópicas e microscópicas estão apresentados nas Tabelas 1 e 2. Das 119 amostras de estômago, 51 amostras $(42,86 \%)$ não apresentaram alterações e 68 amostras $(57,14 \%)$ apresentaram alterações que consistiram em hiperemia de mucosa, úlceras (Fig. 1A), hemorragias petequiais, hipertrofia e atrofia de pregas gástricas (Fig. 1B). His- tologicamente, as alterações mais frequentes em cortes corados pela hematoxilina eosina foram infiltrados de células linfoplasmocitárias, distribuídas na maioria das amostras de forma difusa, por vezes com a presença de folículos linfoides (Fig. 1C), microabcessos e necrose (Fig.1D), hipotrofia epitelial, microabcessose metaplasia epitelial (Fig. 1E). À análise microscópica, em 73,95\% das amostras coradas por Giemsa, foram observadas bactérias helicoidais medindo entre 5 e $12 \mathrm{~mm}$ de comprimento, arranjadas isoladamente ou formando aglomerados no lúmen das glândulas gástricas, na camada de muco superficial ou nointerior das fossetas gástricas (Fig. 1F).

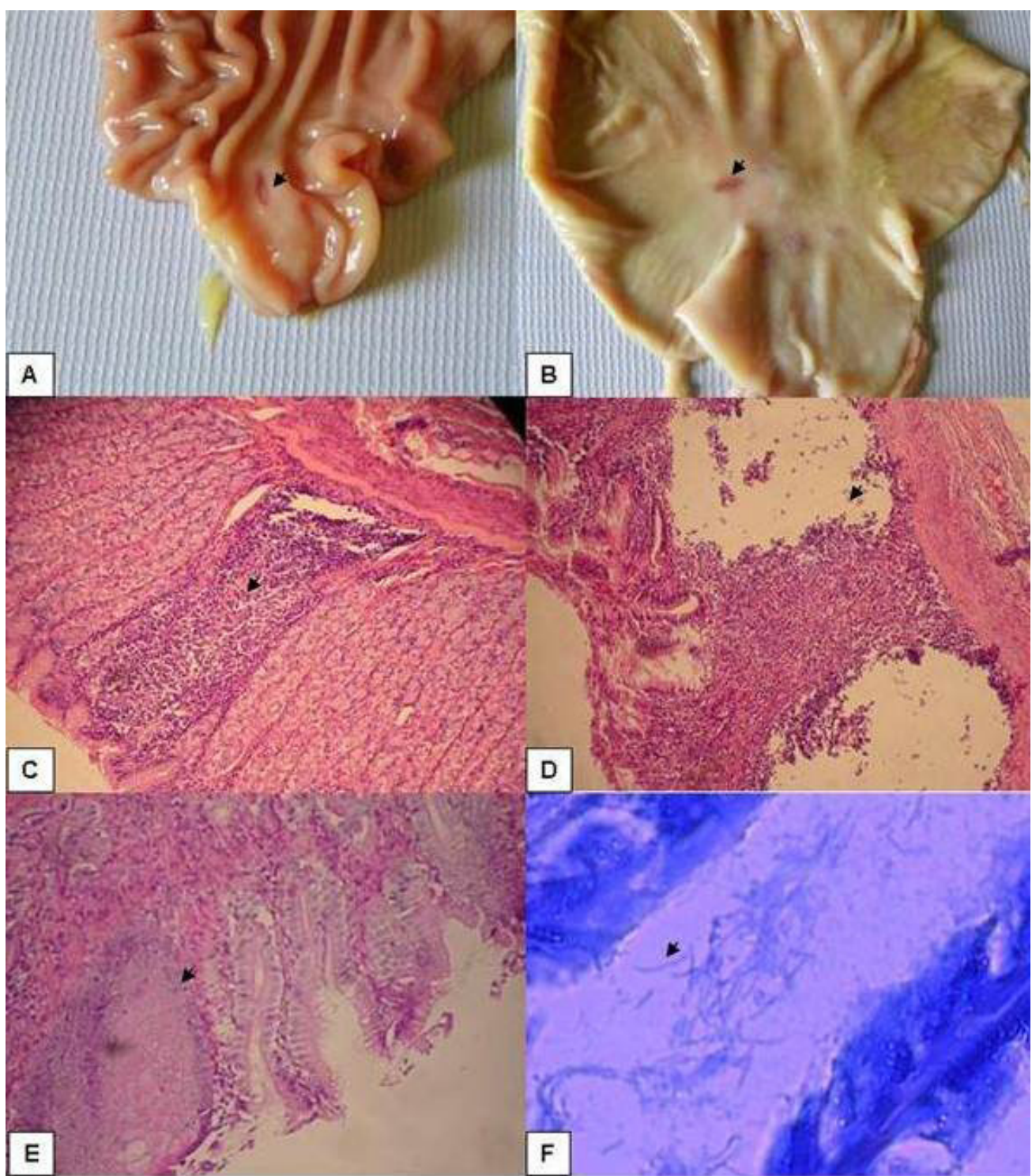

Fig. 1 - (A) Observar úlcera com halo hiperêmico medindo $0,2 \times 0,5 \mathrm{~cm}$ na região pilórica de felino com reação de urease fortemente positiva para Helicobacter spp. (B) Observar áreas de hemorragia e atrofia de pregas gástricas em gato com infecção por Helicobacter spp. (C) Fotomicrografia da mucosa gástrica com presença de folículo linfoide reativo. Coloração H.E. Aumento 280X. (D) Fotomicrografia da mucosa gástrica com presença de infiltrado inflamatório difuso e microabcesso. Coloração H.E. Aumento 280X. (E) Fotomicrografia da mucosa gástrica com infiltrado linfoplasmocitário difuso e metaplasia epitelial. Coloração H.E. Aumento560X. (F) Fotomicrografia da mucosa gástrica mostrando bactérias helicodais medindo $9 \mathrm{~mm}$ na mucosa gástrica pilórica. Coloração Giemsa. Aumento 1.200X. 
Tabela 1 - Incidência de alterações macroscópicas da mucosa gástrica de gatos associadas à infecção por Helibacter spp.

\begin{tabular}{lccc}
\hline \multirow{2}{*}{ Lesões } & \multicolumn{3}{c}{ Região da mucosa gástrica } \\
\cline { 2 - 4 } & Fundo & Corpo & Antropilórico \\
\hline Hiperemia & $02(3,0 \%)$ & $31(45,5 \%)$ & $05(8,38 \%)$ \\
Úlceras & - & $03(4,0 \%)$ & $01(1,88 \%)$ \\
Petéquias & - & $08(11,7 \%)$ & $05(7,3 \%)$ \\
Hipertrofia de pregas gástricas & - & $06(9,24 \%)$ & - \\
Atrofia de pregas gástricas & - & $07(10,9 \%)$ & - \\
\hline
\end{tabular}

Tabela 2. Incidência de alterações microscópicas da mucosa gástrica de gatos associadas à infecção por Helibacter spp.

\begin{tabular}{lccc}
\hline \multirow{2}{*}{ Lesões } & \multicolumn{3}{c}{ Região da mucosa gástrica } \\
\cline { 2 - 4 } & Fundo & Corpo & Antropilórico \\
\hline ILP & $04(3,36 \%)$ & $28(23,52 \%)$ & $12(10 \%)$ \\
ILF-FL & $01(0,84 \%)$ & $08(6,72 \%)$ & $03(2,46 \%)$ \\
ILF-MN & - & $06(5,04 \%)$ & - \\
ILF-HE & - & $04(3,36 \%)$ & $02(1,68 \%)$ \\
ILF-HEN & - & $01(0,84 \%)$ & - \\
ILF-ME & - & $01(0,84 \%)$ & - \\
\hline
\end{tabular}

ILP-infiltrado linfoplasmocitário; ILF-FL-infiltrado linfoplasmocitário difuso com folículos linfoides; ILF-MN-infiltrado linfoplasmocitário difuso com presença folículos linfoides, microabcessos e necrose; ILF-HE-infiltrado linfoplasmocitário com hipotrofia epitelial; ILF-HEN - infiltrado linfoplasmocitário com hipotrofia de epitélio e presença de neutrófilos; ILF-ME - infiltrado linfoplasmocitário com microabcesso e metaplasia epitelial.

Na análise da amostras pelo teste rápido da urease, 98 amostras (82,35\%) apresentaram resultados positivos e 21 amostras $(17,65 \%)$ apresentaram resultados negativos. A reação foi fortemente positiva em 100\% das amostras positivas para este teste.

\section{DISCUSSÃO}

A confirmação diagnóstica dos casos aqui relatados foi baseada nos resultados do teste de urease, lesões macroscópicas características da enfermidade e principalmente na observação de bactérias helicoidais tipo Helicobacter similares aos descritos em outros casos de infecção por Helicobacter spp. em felinos. Os achados de necropsia relatados no presente trabalho são importantes para o diagnóstico presuntivo da doença já que outras bactérias helicoidais podem estar presentes na mucosa gástrica de felinos.

De acordo com Mitchell et al. (2006), na gastrite crônica por Helicobacter spp. visualiza-se edema, hiperemia moderada a acentuada, podendo ainda ocorrer hemorragia (gastrite erosiva hemorrágica aguda) e úlceras. Esses foram achados constantes nos estômagos dos gatos do presente estudo. Atrofia glandular, fibrose e infiltrados linfocíticos são característicos das gastrites, principalmente daquelas que são originadas por $H$. pylori. São, além disso, consideradas como lesões comuns de um processo de infecção, em que existe um desaparecimento progressivo de bactérias e degradação da função gástrica (Tucci et al., 2001). As lesões observadas neste estudo enquadram-se neste padrão histológico.

Lesões neoplásicas estão também relacionadas com a infecção por Helicobacter spp. e particularmente com atrofia do fundo gástrico, estimando-se um risco 4,5 vezes mais elevado de ocorrerem carcinomas nestes casos (Tucci et al., 2001). Os MALTomas também foram relacionados com gastrite atrófica (Hiyama et al., 2001). Esses tipos de alterações não foram identificados nas amostras do presente estudo. No entanto, alterações como infiltração linfoplasmocitária foram identificadas. A formação de linfomas gastrointestinais surgem de processos que geram tecido linfoide associado à mucosa (MALT), que alberga as células precursoras dos linfomas e que, progressivamente, se transforma em células malignas (MOrGner et al., 2000). Assim sendo, e apesar de neoplasias gástricas como o linfoma serem raras em animais, é de alguma relevância investigar a possibilidade de desenvolvimento de MALTomas em gatos com este tipo de lesões histopatológicas.

O teste da urease é preconizado para a confirmação do diagnóstico em casos de helicobacteriose (MARShal; WARREN, 1983; EATON et al., 1996; CASTELLOTE et al., 2001). A técnica é sensível e específica já que todas as espécies de Helicobacter que habitam o estômago são produtoras de urease, apresentandose, portanto, capazes de degradar a ureia em amônia, com consequente mudança do $\mathrm{pH}$ da solução e mudança da tonalidade do meio de inoculação 
(Marshal; Warren, 1983; Eaton et al., 1996; CAStelLOTE et al., 2001).

Utilizando-se do teste da urease e do exame histopatológico pelo método de coloração Giemsa para análise das amostras gástricas dos gatos, obteve-se um elevado resultado positivo em ambos os testes. Essa elevada incidência deve ser levada em consideração na adoção de políticas públicas para a prevenção da infecção por seres humanos. A forma exata pela qual ocorre a transmissão de Helicobacter spp. não é totalmente compreendida. O isolamento de H. pylori em saliva, placa dentária e nas fezes reforça a hipótese de transmissão oro-oral ou orofecal. Alguns relatos mostraram disseminação da bactéria por meio de equipamentos gastrointestinais contaminados em humanos (LANGENBERG et al., 1990; WiLliaMS, 1999).

\section{CONCLUSÃO}

Conclui-se que gatos oriundos da Cidade de Recife, Pernambuco, apresentam alta incidência de infecção por Helicobacter spp. Sendo a presença de infiltrado difuso de células linfoplasmocitárias a alteração histopatológica mais frequentemente observada na mucosa gástrica de gatos infectados.

\section{REFERÊNCIAS}

AVENAUD, P.; MARAIS, A.; MONTEIRO, L.; LE BAIL, B.; BIOLAC SAGE, P.; BALABAUD, CH.; MÉGRAUD, F. Detection of Helicobacter species in the liver of patients with and without primary liver carcinoma. Cancer, v.89, p.1431-1439, 2000.

BARROS, C.S.L.; MARQUES, G.H.F. Procedimentos para diagnóstico das doenças do sistema nervoso central de bovinos. Brasília: Departamento de Defesa Animal, MAPA, 2003. p.50.

BIZZOZERO, G. Sulla presenza di bateri nelle ghiandole gastriche del cane (in itlaian). Atti della Reale Accademia delle Scienze di Torino, v.28, p.249, 1893.

BLASER, M.J. Helicobacter pylori and the pathogenesis of gastroduodenal inflammation. Journal of Infectous Diseases, v.33, p.626, 1990.

BLASER, M.J.; PEREZ-PEREZ, G.I.; KLEANTHOUS, H.; COVER, T.L.; PEEK, R.M., CHYOU, P.H.; STEMMERMANN, G.N.; NOMURA, A. Infection with Helicobacter pylori strains possessing cagA is associated with an increased risk of developing adenocarcinoma of the stomach. Cancer Research, v.55, p.2111-2115, 1995.

BOGLIOLO, L. Patologia. 7.ed. Rio de Janeiro: Guanabara Koogan, 2006.
BOYANOVA, L.; DEREJIAN, S.; KOUMANOVA, R.; KATSAROV, N.; GERGOVA, G.; MITOV, I.; NIKOLOV, R.; KRASTEV, Z. Inhibition of Helicobacter pylori growth in vitro by Bulgarian propolis: preliminary report. Journal of Medical Microbiology, v.52, n.5, p.417-419, 2003.

CASTELLOTE, J.; GUARDIOLA, J.; PORTA, F.; FALCÓ, A. Rapid uréase test: effect of preimmersion of biopsy forceps in formalin. Gastrointestinal Endoscopy, v.53, n.6, p.744, 2001.

CASTRO, L.P. COELHO, L.G.V.; BARBOSA, A.J.A.; VIEIRA, W.L.S. Helicobacter pylori e afeccções associadas. Jornal Brasileiro de Gastroenterologia, v.2, n.3, p.19-30, 2003.

CATTOLI, G.; BART, A.; KLAVER, P.S.J.; ROBIJN, R.J.; BEUMER, H.J.; VAN VUGT, R.; POT, R.G.J.; VAN DER GAGG, I.; VANDENBROUCKEGRAULS, C.M.J.E.;

COVER, T.L.; BLASER, M.J. Helicobacter pylori: a bacterial cause of gastritis, peptic ulcer disease, and gastric cancer. AMS News, v.61, n.5, p.21-26, 1995.

DANESH, J.; YOUNGMAN, L.; CLARK, S.; PARISH, S.; PETO, R.; COLLINS, R. Helicobacter pylori infection and early onset myocardial infarctin: case-control and sibling pairs study. BMJ: British Medical Journal, v.319, n.7218, p.1157-1162, 1999.

EATON, K.A.; DEWHIRST, F.E.; PASTER, B.J.; TZELLAS, N.; COLEMAN, B.E.; PAOLA, J.; SHERDING, R. Prevalence and varieties of Helicobacter species in dogs from random sources and pet dogs: animal and public health implications. Journal of Clinical Microbiology, v.34, n.12, p.3165-3170, 1996.

FOX, J.G.; BATCHELDER, M.; MARINI, R.; YAN, L.; HANDT, L. SHAMES, B.; HAYWARD, A.; CAMPBELL, J.; MURPHY, J.C. Helicobacter pylori-induced gastritis in the domestic cat. Infection and Immunity, v.63, n.7, p.2674-2681, 1995.

GASBARRINI, A.; FRANCESCHI, F. Autoimmune diseases and Helicobacter pylori infection. Biomedicine \& Pharmacotherapy, v.53, n.6, p.223, 1999.

HANDT, L.K.; FOX, J.G.; DEWHIRST, F.E.; FRASER, G.J.; PASTER, B.J.; YAN, L.L.; ROSMIAREK, H.; RUFO, R.; STALIS, I.H. Characterization of Feline Helicobacter pylori Strains and Associated Gastritis in a Colony of Domestic Cats. Journal of Clinical Microbiology, v.33, p.2280-2289, 1995.

HANDT, L.K.; FOX, J.G.; STALIS, I.H.; RUFP, R.; LEE, G.; LINN, J. Helicobacter pylori Isolated from the Domestic Cat: public health implications. Infection and Immunity, v.62. n.6, p.2367-2374, 1994.

HERMANNS, W.; KREGEL, K.; BREUNER, W.; LECHNER, L. Helicobacterlike organisms: histopathological examination of gastric biopsies $\mathrm{f}$ rom dogs and cats. Journal of Comparative Pathology, v.112, p.307-18, 1995. 
HIYAMA, T.; HARUMA, K.; KITADAI, Y.; MIYAMOTO, M.; TANAKA, S.; YOSHIHARA, M.; et al. B-Cell monoclonality in Helicobacter pylori-associated chronic atrophic gastritis. Virchows Archiv, v.438, p.232237, 2001.

JALAVA, K.; ON, S.L.; VANDAMME, P.A.; HAPPONEN, I.; SUKURA, A.; HÄNNINEN, M. L. Isolation and Identification of Helicobacter spp. from Canine and Feline Gastric mucosa. Applied and Environmental Microbiology, v.64, n.10, p.3998-4006, 1998.

LANGENBERG, W., RAUWS, E.A., OUDBIER, J.H., TYTGAT, G.N.J. Patient-to-patient transmission of Campylobacter pylori infection by fiberoptic gastroduodenoscopy and biopsy. Journal of Infectious Disease, v.161, p.507-511, 1990.

MARSHAL, B.J.; WARREN, J.R. Unidentified curved bacilli of gastric epithelium in active chronic gastritis. Lancet, v.1, n.5, p.1273, 1983.

MICHALANY, J. Técnica histológica em anatomia patológica: com instruções para o cirurgião, enfermeira e citotécnico. São Paulo: EPU, 1980. 277p.

MITCHELL, H.; ENGLISH, D.R.; ELLIOTT, F. Immunoblotting using multiple antigens is essential to demonstrate the true risk of Helicobacter pylori infection for gastric cancer. Alimentary Pharmacology \& Therapeutics, v.28, p.903-910, 2006.

MORGNER, A. Malignant tumors of the stomach. Gastric mucosa-associated lymphoid tissue lymphoma and Helicobacter pylori. Gastroenterology Clinics of North America, v.29, n.3, p.593-607, 2000.

MORGNER, A.; LEHN, N.; ANDERSEN, L.P.; THIEDE, C.; BENNEDSEN, M.; TREBESIUS, K.; NEUBAUER, B.; NEUBAUER, A.; STLTE, M., BAYERDÖRFER, E. Helicobacter heilmannii - associated primary gastric low-grade MALT lymphoma: complete remission after curing the infection. Gastroenterology, v.118, p.821-828, 2000.

NEIGER, R.A. Detection and prevalence of Helicobacter infection in pet cats. Journal of Clinical Microbilogy, v.36, n.3, p.634-637, 1998.

NELSON, R.W.; COUTO, C.G. Leucopenia e Leucocitose. In: (Ed.). Medicina interna de pequenos animais. 3.ed. Rio de Janeiro: Elsevier, 2006. p.1187-1190.

NILSSON, H.O.; TANEERA, J.; CAASTEDAL, M.; GLATZ, E.; OLSSON, R.; WASDTRÖM, T. Identification of H. pylori and other Helicobacter species by PCR, hybridization, and particial DNA sequencing in human liver samples from patients with primary sclerosing cholangitis or primary biliary cirrhosis. Journal of Clinical Microbiology, v.38, p.1072-1076, 2000.

NORRIS, C.R.; MARKS, S.L.; EATON, K.A.; TORABIAN, S.Z.; MUNN, R.J.; SOLNICK, J.V. Healthy cats are commonly with "Helicobacter heilmannii" that is associated with minimal gastritis. Journal of Clinical Microbiology, v.37, n.1, p.189-194, 1999.

OTTO, G.; HAZELL, S.H.; FOX, J.G.; HOWLETT, C.R.; MURPHY, J.C.; O'ROURKE, J.L.; LEE, A. Animal and public health implications of gastric colonization of cats by Helicobacter-like organisms. Journal of Clinical Microbiology, v.32, n.4, p.1043-1049, 1994.

QUEIROZ, D.M.; MENDES, E.N.; ROCHA, G.A.; OLIVEIRA, A.M.; OLIVEIRA, C.A.; MAGALHAES, P.P.; MOURA, S.B.; NOGUEIRA, A.M. cagA-positive Helicobacter pylori and risk for developing gastric carcinoma in Brazil. International Journal of Cancer, p.135-139, 1998.

RADIN, M.J.; EATON, K.A.; KRAKOWKA, S.; MORGAN, D.R.; LEE, A.; OTTO, G.; FOX, J. Helicobacter pylori astric infection in gnotobiotic beagle dogs. Infection and Immunology, v.58, n.8, p.2606-2612, 1990.

SOUTO, F.J.D.; FONTES, C.J.F.; ROCHA, G.A.; OLIVEIRA, A.M.R.; MENDES, E.N.; QUEIROZ, D.M.M. Q. Prevalence of Helicobacter pylori Infection in a rural área of the State of Mato Grosso, Brazil. Memórias do Instituto Oswaldo Cruz, v.4, p.93-171, 1998.

TAMS, T.R. Gastroenterologia de pequenos animais. São Paulo: Rocca, 2005.

TUCCI, A.; POLI, L.; BIASCO, G.; PAPARO, G.F.; TOSETTI, C.; FUSAROLI, P.; SAMBRI, V.; DONATI, M.; GRIGIONI, W.; LABATE, A.M.; STANGHELLINI, V.; CALETTI, G. Helicobacter pylori infection and gastric function in patientes with fundic atrophic gastritis. Digestive Diseases and Sciences, v.46, n.7, p.1573-1583, 2001.

VALLE, C.; BIZINELL, S.L.; Prevalência do Helicobacter pylori em pacientes submetidos a endoscopia digestiva Revista Médica do Paraná, v.50, p.17-20, 1993.

WILLIAMS, C.L. Helicobacter pylori and endoscopy. Journal of Hospital Infection, v.41, n.4, p.263-268, 1999.

Recebido em 26/4/11

Aceito em 24/7/12 